\title{
CORRECTION
}

\section{Correction: Antigen-specific regulatory T-cell responses against aeroantigens and their role in allergy}

Petra Bacher ${ }^{1}$ and Alexander Scheffold ${ }^{2}$

Mucosal Immunology (2019) 12:842; https://doi.org/10.1038/s41385-019-0137-5

Correction to: Mucosal Immunology (2018) 11: 1537-50. https://doi. org/10.1038/s41385-018-0038-z; published online 1 June 2018.
The original version of this article contained an error in the published figures, where they appeared in black and white. These have now been corrected to display in colour.

${ }^{1}$ Department of Cellular Immunology, Clinic for Rheumatology and Clinical Immunology, Charité-University Medicine Berlin, Berlin, Germany and ${ }^{2}$ Institute of Immunology, University of Kiel/UKSH Campus Kiel, Kiel, Germany

Correspondence: Alexander Scheffold (alexander.scheffold@uksh.de)

Published online: 31 January 2019 